\title{
Evidencia microbiana de la periimplantitis, factores de riesgo coadyuvantes, diagnóstico y tratamiento según los protocolos científicos
}

\author{
FRANCH F* \\ LUENGO $\mathbf{F} * *$ \\ BASCONES $\boldsymbol{A} * * *$
}

\begin{abstract}
Franch F, Luengo F, Bascones A. Evidencia microbiana de la periimplantitis, factores de riesgo coadyuvantes, diagnóstico y tratamiento según los protocolos científicos. Av Periodon Implantol. 2004; 16, 3: 143-156.
\end{abstract}

\begin{abstract}
RESUMEN
Se presenta un trabajo de revisión sobre periimplantitis, comenzando con el desarrollo de los conceptos básicos de la anatomía periimplantar y los criterios de osteointegración, se hace un estudio sobre la evidencia microbiológica de la patología periimplantaria y la patogenia de la misma, conjuntamente con los factores de riesgo que afectan al proceso inflamatorio y destructivo de los tejidos periimplantarios. Continua el trabajo exponiendo los parámetros clínicos que muestra la enfermedad, como desarrollar el diagnostico y que posibilidades terapéuticas y de mantenimiento basadas en los protocolos científicos se pueden aplicar.
\end{abstract}

\section{PALABRAS CLAVE}

Periimplantitis, Infección periimplantaria, Factores de riesgo, Tratamiento de la periimplantitis

Aceptado para publicación: Abril 2004

\section{INTRODUCCIÓN}

Con la irrupción de la implantologia en la práctica de la odontoestomatologia, la osteointegración, se ha convertido en un método para le rehabilitación de pacientes desdentados total o parcialmente.

Durante los últimos años, se ha demostrado con estudios, los resultados a largo plazo de la integración tisular y ósea de los implantes dentales $(1 ; 3 ; 13 ; 29 ; 33 ; 47)$

A pesar de los resultados satisfactorios, los tejidos que soportan los implantes osteointegrados son susceptibles a patologías que pueden llevar a la pérdida del implante $(23 ; 40)$
Diversos factores de riesgo aparecen detrás de dichas situaciones, con lo que debemos valorar, hábitos tabáquicos,(8) calidades óseas (32) factores sistémicos, riesgos ocasionados por trauma quirúrgico y contaminación bacteriana durante la inserción, o incluso una mala distribución de fuerzas que generen sobrecarga. Todos estos últimos tópicos, están relacionados con la perdida prematura del implante. En cambio, hay factores que se relacionan más, con la pérdida tardía de los implantes. En ellos, se ven involucrado el medioambiente de la cavidad oral y la capacidad del propio individuo para mantener un equilibrio con el mismo. Son parámetros similares a las lesiones periodontales asociadas a dientes y están íntimamente relacionadas con la carga microbiana de la placa bacteriana.

* Licenciado en Odontología por la Universidad Internacional de catalunya.

** Secretario general de la SEPA. Práctica privada exclusiva en periodoncia e implantes

*** Catedrático de medicina bucal y periodoncia. Universidad Compluténse de Madrid. 
En este artículo no debemos confundir la perdida prematura del implante con él termino de periimplantitis, que se definiría como un proceso inflamatorio que afecta a los tejidos que rodean al implante osteointegrado en función, con la consecuente pérdida de hueso de soporte y de la osteointegración. (4)

Con el objetivo de revisar los factores de riesgo, los parámetros diagnósticos utilizados para definir periimplantitis, los posibles planes de tratamiento y mantenimiento de los implantes osteointegrados, es importante conocer la morfología de los tejidos periimplantarios y los criterios de éxito y fracaso de los implantes.

\section{MORFOLOGÍA DE LOS TEJIDOS PERIIMPLANTARIOS}

Los tejidos blandos que rodean al implante son muy semejantes en su estructura y composición a los tejidos periodontales. El tejido supracrestal que rodea los implantes se denomina mucosa perimplantaria y forma en torno al implante el surco periimplantario. Este tejido esta recubierto en su vertiente interna por el epitelio del surco y el en la parte más apical del mismo se continua con las células del epitelio de unión. En su vertiente externa esta recubierto por el epitelio oral que puede ser queratinizado o simple mucosa alveolar. Entre las células más apicales del epitelio de unión y el hueso alveolar se encuentra la zona del tejido conectivo que entra en contacto directo con la superficie del implante.

Epitelio del surco: es una extensión no queratinizada del epitelio oral y constituye la pared más externa del surco perimplantario (Listgarten y col 1975). En el surco periimplantario se produce de igual manera que en el surco periodontal fluido crevicular que con- tiene proteínas del complemento, enzimas, e inmunoglobulinas. (16)

Epitelio de unión: Al igual que en los dientes se une a la superficie de los implantes a través de le lamina basal y de hemidesmosomas. Las células más apicales del epitelio de unión están a 1-1,5 mm de la cresta ósea alveolar. Esta zona es por tanto de extrema importancia y un punto crítico, pues supone el sellado biológico a las sustancias exógenas. Si Este sellado se destruye, las fibras más apicales del epitelio de unión migraran, dado que no existe cemento que recubra la superficie del implante ni fibras a su alrededor que frenen el proceso destructivo.(2)

Epitelio ginigival oral: podemos clasificar dos tipos, mucosa masticatoria y mucosa alveolar. Muchos autores prefieren a ser posible que la mucosa que rodee al implante sea mucosa masticatoria por su mayor resistencia, pero diversos estudios han demostrado que no existen diferencias en cuanto al control de placa y a la salud de la mucosa periimplantaria entre lugares con o sin una adecuada mucosa masticatoria.(6)

Tejido conectivo periimplantario: Entre las estructuras epiteliales y el hueso alveolar hay una zona de tejido conectivo que también entra en contacto directo con la superficie del implante, observándose fibroblastos, unidos mediante una capa de glucoproteinas a la capa de oxido de titanio. Tiene mayor proporción de colágeno y menor cantidad de fibroblastos, que su homologa en el periodonto, y esta surcada por haces de fibras que circulan paralelas a la superficie del implante, originando un manguito fibroso periimplantario que le da consistencia y tonicidad a la mucosa.(10) No aparecen fibras de características equivalentes a las dentogingivales, dentoalveolares y transeptales, por lo que la labor de inhibición de la migración apical de la adherencia epitelial queda en manos de la interacción entre el conectivo y el oxido de titanio.(20)

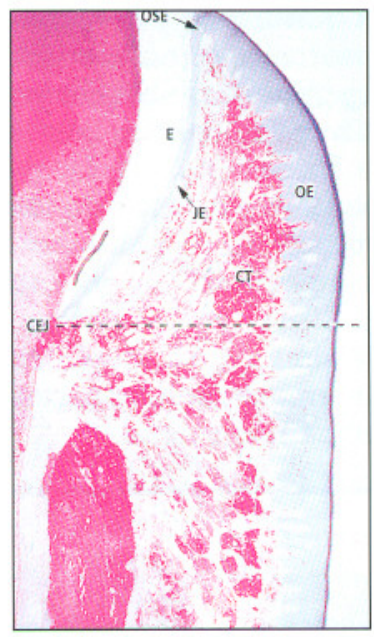

Fig. 1
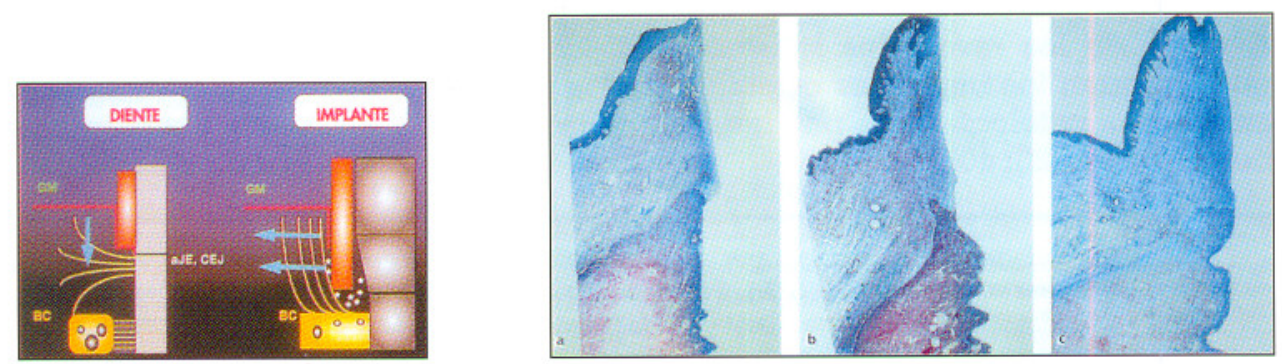
Hueso perimplantario: En un principio Schroeder y col 1976 definió la unión del hueso al implante como una anquilosis funcional. Luego llega el concepto de la osteointegración, pronunciado por Branemark, el cual la define como una conexión estructural y funcional entre el hueso vivo y la superficie del implante que soporta una carga. Una serie de estudios histológicos trataran de evaluar la conexión del hueso al implante su estructura y composición.

- Células óseas periimplantarias: en la interfase titanio hueso cortical se encuentran osteocitos que a través de sus prolongaciones citoplasmáticas se acercan al titanio. En el tejido esponjoso se distinguen trabeculas oseas, osteoblastos, fibroblastos y estructuras vasculares cerca del oxido de titanio que recubre al implante.

- Capa de proteoglicanos: Es una capa de 200 A de espesor de sustancia fundamental amorfa que se encuentra parcialmente calcificada alrededor del implante.

- Filamentos de colágena: Separada por la capa de proteoglicanos, aparece en la interfase del implante haces de colágeno a una distancia de 1-3 $\mu \mathrm{m}$. Las fibras de colágena que se disponen en líneas paralelas y se encuentran adheridas al titanio. (Fig. 1)

\section{CRITERIOS DE ÉXITOY DE LOS IMPLANTES OSTEOINTEGRADOS}

Existen discrepancias a la hora de definir dichos criterios ya que en ocasiones están muy relacionados al tipo de implante o al sistema utilizado, pero de modo generalizado se atribuye bajo criterio internacional aceptar los postulados por Albrektsson T.(5) en 1986 aunque parece que deban tenerse en cuenta una serie de indicaciones complementarias expuestas por Zarb y Marc Bret.

\section{Criterios de éxito de Albrektsoon:}

- Un implante aislado e independiente debe ser inmóvil cuando se prueba clínicamente

- La radiografía no debe de mostrar ninguna zona radiolúcida alrededor del implante

- La perdida ósea vertical anual debe de ser inferior a $0,2 \mathrm{~mm}$ después del primer año de la puesta en función del implante.

- Cada implante debe de estar libre de sintomas persistentes y/o irreversibles como dolor, infecciones, neuropatias, parestesias, o lesión del conducto mandibular.

- Un mínimo porcentaje de éxito del $85 \%$ y del $80 \%$ a los 5 y 10 años respectivamente debe de ser reportado bajo los criterios expuestos anteriormente.

Zarb comparte los criterios de Albrektsson, matizando, que los implantes deben cumplir con un objetivo y una finalidad clara de satisfacer tanto estética, como funcionalmente, al paciente y que por ello se debe considerar muy seriamente el desarrollo de la prótesis que posteriormente soportaran dichos implantes.

\section{CONCEPTOS DE MUCOSITIS Y PERIIMPLANTITIS}

En el Workshop europeo de periodontología celebrado en febrero de 1993 (Suiza) por la Federación Europea de Periodontología, se acuñaron denominaciones para dos patologías inflamatorias periimplantarias bien diferenciadas: mucositis periimplantaria y periimplantitis.

La enfermedad periimplantaria se caracteriza por la inflamación de los tejidos periimplantarios y puede manifestarse de las siguientes formas:

- Mucositis periimplantaria: Como formas reversible de afección inflamatoria de los tejidos blandos que rodean a un implante en función.

-Periimplantitis: Forma irreversible de afección inflamatoria de los tejidos blandos que rodean al implante en función, en la que además se produce una pérdida ósea.

\section{ETIOLOGÍA DE LA PERIIMPLANTITIS}

A pesar de las elevadas tasas de éxito de los implantes, los fracasos ocurren y es importante establecer la etiología, la patogénesis, diagnóstico, prevención y plan de tratamiento a seguir.

Lindhe describen en 1992 que las lesiones en los tejidos blandos alrededor de los implantes son potencialmente más peligrosas que con respecto a los dientes ya que tienden a extenderse apicalmente con mayor facilidad hacia el tejido óseo periimplantario.

El fracaso de los implantes puede ocurrir en dos fases, que debemos tener bien diferenciadas. 
La primera fase o la perdida prematura del implante, se producirá cuando este aun no haya llegado ha osteointegrarse a consecuencia de diversos factores de riesgo que consideraremos y que más adelante expondremos más detalladamente, tales como pobre estabilidad primaria, contaminación bacteriana, enfermedades sistémicas, hábitos tabáquicos, mala técnica quirúrgica, mala calidad ósea, etc.

La segunda fase o etapa en la que puede producirse la perdida del implante es cuando este ya esta osteointegrado y en función.

Actualmente se piensa que el fracaso de los implantes después del proceso de osteointegracion esta principalmente motivado por la infección bacteriana. A pesar de la posibilidad expuesta por diversos autores que lo justifican por la sobrecarga oclusal (31) pero no se ha establecido evidencia científica que lo avale, tal y como demostró (36).

La mayoría de los estudios prospectivos y retrospectivos de supervivencias de implantes utilizando implantes ITI demostraron que un muy reducido número de fracasos se podría asociar a sobrecarga oclusal (13-15; 19) apuntando a consecuencia de estos que :

- La tasa de éxito de los implantes ITI tras 5 y 8 años de funcionamiento es igual o mayor a la de otros sistemas y que la mayor causa de fracaso se atribuyen a infecciones periimplanntarias.

- En estudios a largo plazo los pacientes con una higiene adecuada mantienen los implantes mas tiempo en condiciones estables y de salud.

- En estudios a largo plazo no hay diferencias estadísticamente significativas entre los implantes de $8 \mathrm{~mm}$ y los de 10 y $12 \mathrm{~mm}$ de longitud.

\section{MICROBIOLOGÍA ASOCIADA A PERIIMPANTITIS}

La flora bacteriana en la cavidad oral antes de la colocación de implantes osteointegrados va a determinar la composición de la nueva microbiota que se va a formar alrededor de los mismos. Muchos investigadores han intentado relacionar el biofilm bacteriano inducido alrededor de los implantes con el biofilm dental encontrado en la enfermedad periodontal (62).

Es importante considerar la situación en la que se encuentra el paciente previa rehabilitación con implantes, debemos distinguir a aquellos que son desdentados totales de los que son parcialmente edéntulos.
- Cuando no existe ningún tipo de patología la flora esta compuesta por cocos gram positivos, aerobios y bacilos inmóviles, tanto en implantes como en dientes (18)

- En situaciones patológicas la flora tanto en dientes como en implantes estará compuesta por bacterias anaerobias, gram negativas y encontraremos también aumentado el porcentaje de bacilos móviles, fusiformes y espiroquetas (Prevotella intermedia, Fusobacterium nucleatum, Porphyromonas gingivalis, Capnocytophaga, etc.) demostrado en diversos estudios (22; 57 ; $58 ; 62 ; 64)$

\section{Evidencia de la etiología microbiana de las infecciones periimplantarias}

Muchos factores contribuyen al fracaso de los implantes, sin embargo un numero creciente de estudios demuestran que es evidente el efecto negativo de la presencia de bacterias anaeróbicas en los tejidos periimplantarios. Existen 5 líneas de evidencia que soportan la idea del papel fundamental de los microorganismos en la etiología de la periimplantitis:

\section{1- Los depósitos de placa en implantes puede inducir mucositis periimplantaria}

Tal y como se demostró e el modelo clásico de gingivitis experimental descrito por Löe en 1965 que representa la prueba final de la relación causa efecto entre el acumulo de placa y la gingivitis. Se ha podido repetir para los casos de infecciones periimplantarias (56). Tras un periodo de control de placa durante 6 meses en pacientes con los implantes ya cargados se inhibió la higiene durante 3 semanas. El resultado de acumulo de placa se tradujo en un aumento de la inflamación y de la profundidad del sondaje alrededor de los implantes, demostrando así la relación entre la del acumulo de placa y el desarrollo de la periimplantitis. Por otro lado un estudio realizado por Berglundh T ,en 1992 (11) manifiesta la respuesta tisular a la presencia de placa en perros beagle desarrollándose un infiltrado inflamatorio en cantidad igual que el de los dientes adyacentes, que indica una respuesta inicial del hospedador en la mucosa periimplantaria igual a la que ocurren en la encía.

\section{2- La demostración de diferencias cuantitativas y cualitativas en la microflora asociada con éxito y fracaso de los implantes. Factor de asociación}

Las situaciones de éxito y fracaso de implantes presentan diferencias marcadas en la composición de la 
flora asociada. La flora bacteriana que coloniza los implantes exitosos esta constituida por cocos gram positivos, mientras que en los casos de de fracaso de implantes se encuentran bacterias gram negativas anaerobias tales como Porfiromonas gingivalis, Prevotella intermedia, Fusobacterium nucleatum, y Actinomices actinomicetemcomitans (58), fusobacterias y espiroquetas (7).Los estudios longitudinales han demostrado que la cantidad de bacterias presentes en los casos de éxito de implantes es baja y la composición de la flora no cambia respecto a la situación normal.(12) Autores diversos señalan que en el caso de pacientes parcialmente desdentados con peridontitis previa que van a ser rehabilitados con implantes puede suceder que los microorganismos periodontopatógenos que se encuentran en el biofilm dental sean transmitidos desde dichos dientes remanentes a los implantes.

Por otro lado los estudios microbiológicos indican una marcada diferencia en la composición de la flora periimplantaria entre implantes con surcos o bolsas profundas que aquellos que carezcan de ella y en los que la profundidad sea menor $(43 ; 60)$.

\section{3- Colocación de ligaduras en animales generando así una alteración de la composición de la microflora y periimplantitis}

Estudios experimentales en animales han permitido inducir periimplantitis a través de la colocación de ligaduras que conducen a un rápido acumulo de placa $(35 ; 64)$ induciendo así periimplantitis y periodontitis en monos, demostrándose una agudización de los parámetros clínicos de inflamación y pérdida de inserción y alteraciones histológicas y microbiológicas.

\section{4- La terapia antimicrobiana mejora los parámetros clínicos de los pacientes con periimplantitis}

Muchos estudios han analizado el potencial de antimicrobianos sistémicos y tópicos con el tratamiento de infecciones periimplantarias. La administración de sistémica de amoxicilina y metronidazol en perros asociada a desbridamiento local permite la resolución de periimplantitis inducida (21). También parece favorecer la situación de patologia periimplantaria, la colocación de fibras de tetraciclina. (61).

\section{5- El nivel de higiene tiene un impacto en el éxito a largo plazo del tratamiento con implantes}

Los pacientes con inadecuada técnica de higiene oral, presentan mayor reabsorción ósea alrededor de los implantes (39).Esta evidencia implica el hecho de que un buen mantenimiento de los pacientes rehabilitados con implantes tiene como objetivo eliminar los depósitos bacterianos, evitar la colonización de la bacterias y alterar la ecología del biofilm alrededor de los implantes de forma que se impida la multiplicación de los patógenos potenciales.

\section{FACTORES DE RIESGO QUE AFECTAN A LA PÉRDIDA PREMATURA DE IMPANTES OSTEOINTEGRADOS}

\section{Contaminación preoperativa}

Una posible contaminación directa de bacterias durante el procedimiento quirúrgico a través de guantes, aire contaminado, saliva, instrumentos mal esterilizados, pueden cursar con la contaminación del implante o del lecho y generarse un absceso periapical (53-55).

Lecho receptor del implante contaminado o infectado

Procesos infecciosos, inflamatorios muy próximos a la zona de inserción del implante, tales como lesiones periapicales, quistes restos radiculares o cuerpos extraño como restos de procedimientos endodónticos pueden interferir en la osteointegración (63).

\section{Irritantes como el tabaco.}

En pacientes fumadores existe un mayor riesgo de periimplantitis que en los no fumadores ya que el tabaco actúa como irritante e interfiere en el sistema inmunitario de defensa, lo que agrava cualquier lesión periodontal o periimplantaria.

El tabaco incrementa el riesgo de fracaso implantológico en 11,5 a 2,5 veces más (41).

\section{Enfermedades sistémicas}

Las enfermedades sistémicas que se asociaran a la periimplantitis se dividirián básicamente en las alteraciones secundarias que comprometen la inmunidad del huésped, como por ejemplo las leucemias y el SIDA; y las alteraciones endocrinas y metabólicas como la diabetes y el embarazo.

Y particularmente aquellos pacientes que afectados por osteoporosis vean comprometida su calidad ósea para la correctas implantación de los implantes dentales.

\section{Tipo de superficie del implante}

Los implantes tratados con ácidos los rociados con plasma spray de titanio y los recubiertos por hidro- 
xiapatita son nichos perfectos para la colonización bacteriana, Además con el aumento de la porosidad de la superficie de los implantes hay una mayor dificultad de higienización y de mantenimiento de la salud periodontal $(57 ; 62)$.

\section{Fallos por la técnica quirúrgica}

Recalentamiento del hueso en el momento del fresado y de la inserción del implante.

Compresión excesiva del hueso, hay profesionales que refieren que buscar una estabilidad primaria a costa de insertar implantes a más de 35-40 Nm implica un riesgo de necrosis por la tensión generada sobre el hueso circundante al implante.

\section{No adecuada estabilidad primaria}

Una vez insertado el impalante este no quede estable y se produzcan micro movimientos que cursen con la ausencia de osteointegración y, por el contrario, con una cicatrización con tejido fibroso. Esta situación es mas frecuente en huesos blandos y de mala calidad. Factor asociado probablemente a la pérdida por sobrecarga.

\section{PATOGENIA DE LA PERIIMPLANTITIS}

Las bacterias de la cavidad oral se acumulan en los tejidos periimplantarios y desencadenan en ellos una reacción inflamatoria. Provocan daño tisular mediante diversos mecanismos:

- Toxicidad de sus propios componentes: Como las endotoxinas o los lipopoliscáridos, o con producción de sustancias nocivas como colagenasas, fosfatasas ácidas, fosfolipasas, fosfatasas alcalinas y proteasas.

- Estimulación de la inmunidad Humoral y celular: Con activación de los macrófagos, leucocitos polimorfonucleares Linfocitos $\mathrm{T}$ y celulas plasmaticas que mediante la reacción proinflamatoria estimulan procesos de destrucción de tejidos periimplantarios (49).

Como consecuencia del acumulo de placa bacteriana tanto las bacterias como sus productos atraviesan la barrera mucosa que tiene función de sellado. A partir de este momento, se inicia el proceso inflamatorio que cursa con la destrucción del colágeno y el hueso alveolar. Los mecanismos de destrucción ósea se caracterizan por la inflamación y la actividad osteoclástica que va a provocar la reabsorción del hueso que se encuentra en contacto íntimo con el implante.

\section{CLÍNICA}

La periimplantitis se puede observar clínicamente por:

- La presencia de placa bacteriana y calculo alrededores del implante.

- Por un edema y enrojecimiento de tejidos marginales.

- Hiperplasia de la mucosa en las zonas donde no hay demasiada encía queratinizada.

- Aumento de la profundidad de sondaje y ligero sangrado y o supuración tras el mismo.

- Destrucción ósea vertical en relación con la bolsa periimplantaria.

- Presencia radiológica de reabsorción ósea.

- Movilidad del implante.

- Persistencia de dolor y mal estar.

La movilidad y radiolucidez periimplantaria contínua, indica que esta próximo el desenlace final de la enfermedad caracterizado por una perdida total de la interfase entre el hueso y el implante.

Los signos clínicos que indican que el fracaso del implante es por sobrecarga oclusal son, la ausencia de inflamación y de bacterias gram negativas, estando los demás signos anteriormente mencionados, presentes.

\section{FRECUENCIA DE LA PERIIMPLANTITIS}

Es difícil estimar la frecuencia de periimplantitis debido a los distintos criterios utilizados para discernir entre salud y enfermedad, el baremo estipulado va de un $0,8 \%$ a un $11 \%$.

\section{DIAGNÓSTICO DE PERIIMPLANTITIS}

El diagnóstico precoz en implantologia es un momento fundamental para la intercepción y tratamiento de un estadio patológico inicial, aunque detectar zonas potenciales de enfermedad es complejo.

Una lesión de periimplantitis avanzada es fácilmente diagnosticable a través de radiografías detectando una perdida ósea alrededor del implante, incluso es ya evidente la situación crítica, cuando existe movilidad del implante. Por ello los procedimientos de diagnóstico deben ser utilizados a modo de: 
- Identificar la enfermedad periimplantaria.

- Proceder al diagnóstico diferencial entre mucositis y periimplantitis.

- Planear el tratamiento que puede incluir, instrumentación mecánica, procedimientos regenerativos, colocación de antisépticos locales, la administración de antibióticos sistémicos y la destoxificación de la superficie del implante.

- Evaluar el resultado del tratamiento y establecer un buen programa de mantenimiento.

\section{Parámetros de diagnóstico clínico}

Se recomienda un examen periódico anual, en el se debe realizar:

\section{Examen clínico(fig 2)}

- Desmontar la prótesis para poder verificar el estado de salud de los tejidos periimplantarios.

- Comprobar la higiene y la existencia de tártaro en los pilares transmucosos.

- Inspección y palpación de los tejidos blandos periimplantarios, para detectar posibles signos de inflamación, sangrado, exudado o supuración.

Sondaje: con un instrumento plástico milimetrado, alrededor del implante en los casos donde la morfología protésica lo permita, valorando:
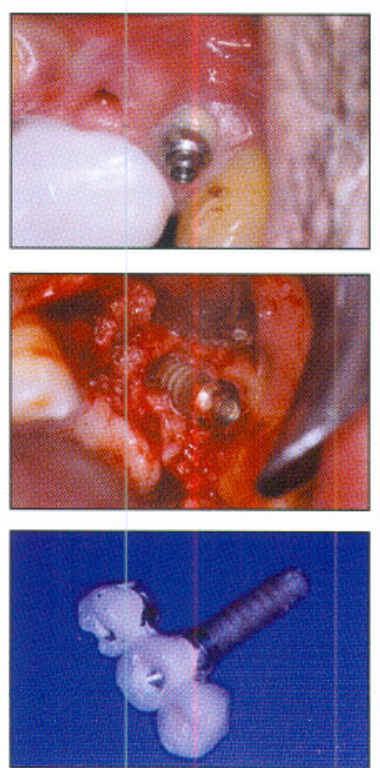

- La profundidad de sondaje periimplantaria, Anotando que La mayoría de los estudios indican una penetración de la sonda periodontal de $3 \mathrm{~mm}(1 ; 20)$. Existe una relación entre el nivel de hueso que aparece en las radiografías y la penetración de la sonda periodontal (15) Anotando que la discrepancia entre la penetración de la sonda y la localización del margen óseo en las radiografías es de $1,17 \mathrm{~mm}$ en implantes no sumergidos.

- La distancia entre el margen del tejido blando y una referencia en el implante, valorando así las recesiones o las hipertrofias.

- Sangrado al sondaje y la supuración desde el espacio periimplantario.

Siendo el sangrado al sondaje una reacción de los tejidos blandos, es importante matizar que el sangrado al sondaje no tiene porque prever actividad de la enfermedad, pero la ausencia de sangrado es utilizado clínicamente como indicador de estabilidad periodontaltal y como estudió Lang 1990

Por otro lado la supuración en las evaluaciones histológicas de los tejidos periodontales demostraron la infiltración de neutrófilos en los casos de enfermedad en lo estudios desarrollados por Schcroeder y colaboradores 1991. La supuración indica actividad de la enfermedad y requiere de tratamiento antinfeccioso.

Si durante el examen del implante se advierte La movilidad del mismo esto indica ausencia o falta de osteointegración. Es un parámetro tardío y nos traslada a una decisión de remoción del implante.

\section{Examen radiológico (fig. 3)}

Uno de los elementos más importantes de la revisión clínica es la valoración del tejido óseo periimplantario, para ello recurrimos al examen radiológico. Pese a que las radiografías solo muestran la situación ósea mesial y distal, se constata que los defectos óseos tienen forma circular o de embudo y por tanto son mayores de lo que observamos en una radiografía (49).

La forma del defecto óseo es muy importante como valor pronóstico y posterior tratamiento (49). Diferenciamos entre defectos Horizontales y verticales.

Defectos horizontales: Su desarrollo es lento y se asocian con una retracción de las partes blandas, el ángulo que forma con la superficie del implante es mayor de 60 grados. Este tipo de defectos suelen tener un pronóstico más favorable.

Defectos verticales: Se desarrollan mas rápidamente y se producen
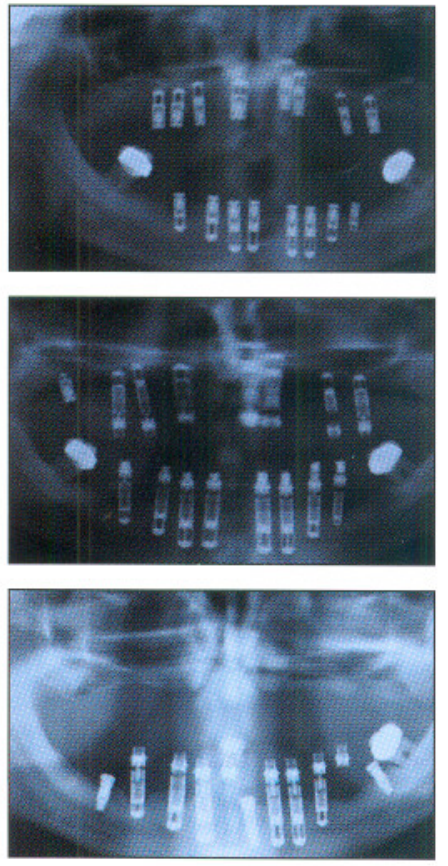
bolsas con proliferación epitelial en su interior, el ángulo que se forma con la superficie del implante es menor de 60 grados. Suelen producirse infecciones purulentas cuando la profundidad de sondaje es mayor de $5 \mathrm{~mm}$.

Por otro lado Jovanovic clasificó los defectos óseos de forma que se valorase la morfología del defecto, su tamaño para determinar a posteriori una adecuada acción terapéutica. Se definieron 4 grados de defectos:

-Grado 1: Existe una perdida ósea horizontal mínima con signos iniciales de reabsorción vertical periimplantaria.

-Grado 2: Pérdida ósea horizontal moderada con reabsorción vertical periimplantaria localizada.

-Grado 3: Pérdida ósea horizontal moderada-intensa con reabsorción vertical circumferencial avanzada.

-Grado 4: Pérdida ósea horizontal intensa con reabsorción vertical circunferencial avanzada y pérdida vestibular o lingual.

\section{Examen clínico microbiológico}

Existen pruebas diagnósticas para detectar determinados patógenos. El cultivo microbiológico es uno de los mas frecuentes y se puede realizar mediante una muestra del exudado periimplantario tomado con puntas de papel estériles (57).

Es aconsejable realizar un antibiograma para conocer la sensibilidad antibiótica de la flora microbiana subgingival periimplantaria.

También existen métodos indirectos que muestran la presencia bacteriana en el fluido del surco periimplantario. Recientemente se han desarrollado sondas de DNA para identificar las secuencias de nucleótidos especificas de determinadas especies bacterianas. También se pueden detectar especies bacterianas seleccionadas mediante la búsqueda de enzimas únicas, correspondientes a una o más especies clínicamente relevantes. (18)

\section{TRATAMIENTO DE LA PERIIMPLANTITIS}

La frecuencia de fracasos implantarios es relativamente baja y el número de estudio longitudinales que evalúan los distintos protocolos de tratamiento de periimplantitis es limitado.
Por definición la periodontitis, como ya hemos tratado de demostrar, conlleva una naturaleza inflamatoria y etiología bacteriana que cursa con la pérdida del soporte óseo periimplantario. Por lo tanto desde un punto de vista etiopatogénico, existen dos niveles de tratamiento. La etiología infecciosa y posteriormente el tratamiento de los defectos óseos secundarios a dicha infección.

Los objetivos que debe de cumplir nuestro tratamiento son $(44 ; 46)$

-Eliminación de la placa bacteriana de la bolsa periimplantaria

-La decontaminación y acondicionamiento de la superficie del implante

-La reducción o eliminación de zonas que no pueden ser mantenidas sin placa salvo mediante correctas técnicas de higiene oral

-Establecimiento de un control de placa eficaz para la prevención de mucositis y reinfección.

-Regeneración del hueso periimplantario perdido en el curso patológico de la enfermedad (fig 4)

\section{Tratamiento de la periimplantitis mediante terapia antiinfecciosa}

La terapia antiinfecciosa incluye las técnicas de desbridamiento de las lesiones, la descontaminación del implante y el empleo de una correcta antibioticoterápia sistémica y o en su defecto, antisépticos locales.

La irrigación subgingival del espacio periimplantario con agentes antisépticos es un tratamiento utilizado por muchos clínicos.(17) en un estudio doble ciego, evaluaron 20 casos de periimplantitis en el que la utilización de enjuagues con antisépticos redujo el índice de placa , y el índice gingival. (37)demostraron que la irrigación con clorhexidina no produce efectos clínicos ni microbiológicos en casos de periimplantitis de implantes recubiertos por hidoxiapatita.

Según Ericsson en 1996(21) en un estudio en el que se genero periimplantitis, mediante ligaduras en perros labrador sobre implantes Branemark la combinación de antimicrobianos sistémicos tal como el metronidazol y la amoxicilina acompañados de un correcto desbridamiento mecánico conlleva a la resolución de las lesiones periimplantarias, una recesión de los tejidos circundantes y una modificación en la base del defecto óseo.

Persson y col 1999(50) obtuvieron similares resultados en perros Beagle, y el tratamiento consistió en amoxicilina y metronidazol durante tres semanas junto con desbridamiento quirúrgico local y detoxificacción de la superficie del implante con piedra pomez y solución salina. 

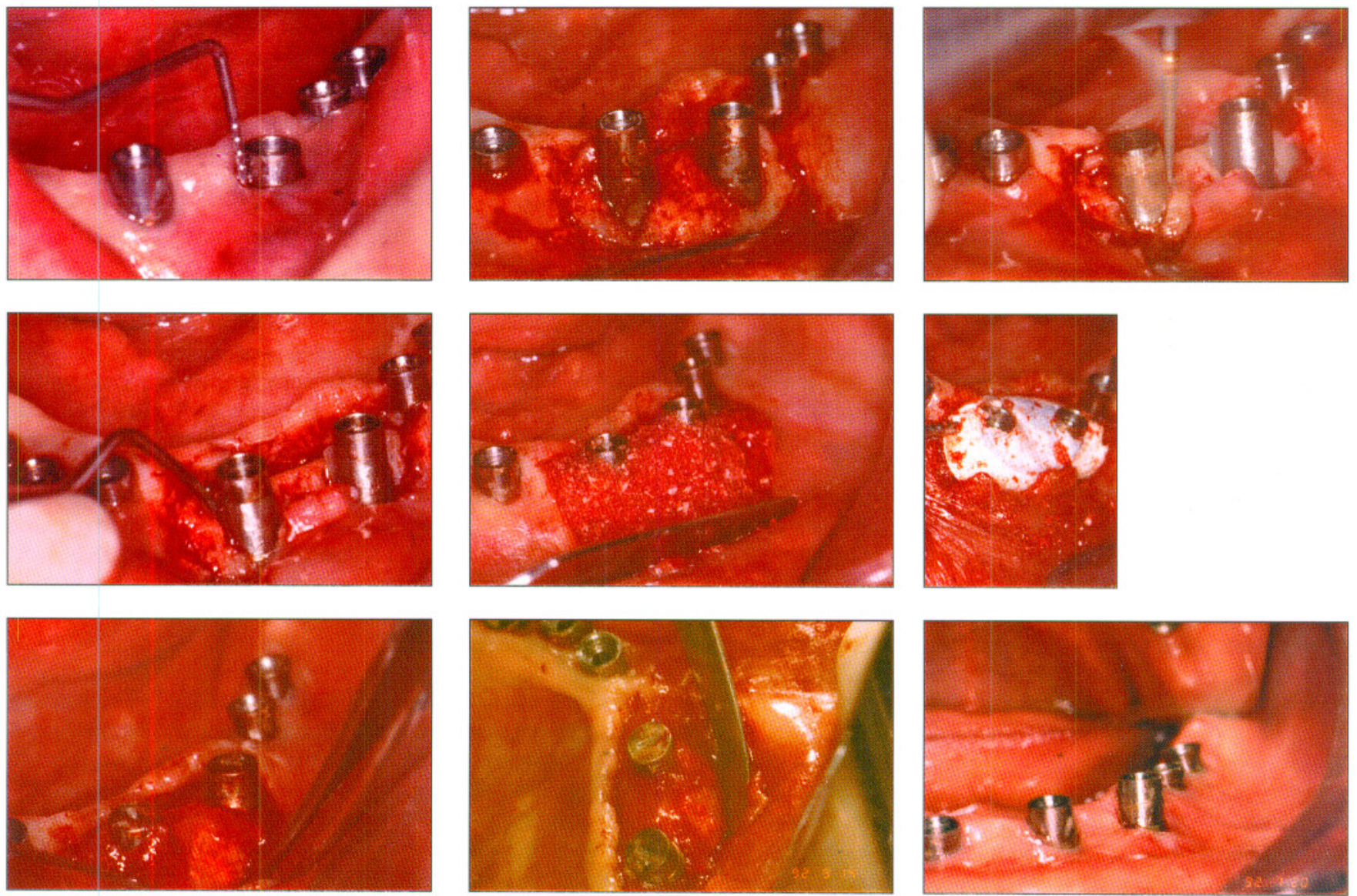

Fig. 4

Mombelli y col 1992 (45) realizaron tratamiento antimicrobiano en 9 pacientes con perdida ósea severa alrededor de implantes ITI. La pauta de tratamiento consistía en desbridamiento mecánico seguido de irrigación de las bolsas periimplantarias con profundidad de sondaje superior a 3mm con CHX 05\% y administración sistémica de $\mathrm{lg}$ de Ordinazol durante un periodo de diez días. Se produjeron mejorías en los parámetros clínicos aunque los datos microbiológicos demostraron cierta recidiva en la composición de la microbiota, Lo que cuestiona la efectividad del tratamiento.

Schenk y col 1997 (61) propusieron la aplicación local de agentes antimicrobianos de liberación controlada para el tratamiento de la periimplantitis, utilizaron fibras de tetraciclinas con resultados satisfactorios para el tratamiento de las mucositis e hipertrofias alrededor de los implantes.

Weber en 1994 en un modelo animal utilizaron los aines flurbiprofeno con el objetivo de disminuir la pérdida ósea. Los resultados dieron una perdida ósea en el grupo placebo mientras que en aquellos que se les había administrado el aine no se denoto perdida de soporte.

\section{Tratamiento de la superficie del implante}

Cuando un implante pierde osteointegracion la superficie de titanio queda expuesta a la cavidad oral y cubietra por un biofilm. Esto lleva a la formación de una biopelícula en la superficie, seguida de colonización bacteriana.

La cantidad y maduración de la placa bacteriana que cubre la superficie del implante es mayor en surperficies rugosas $(34 ; 59)$ y sugieren el pulido de la porción expuesta para prevenir reinfección y facilitar los procedimientos de higiene oral.

La decontaminación de la superficie de los implantes expuestos es fundamental cuando se utilizan procedimientos regenerativos para obtener nuevo hueso alrededor de implantes con periimplantitis (27). Se han utilizado distintas técnicas tales como ácido cítrico, tetraciclina y hcl (61), arenado, arenado y ácido cítrico, láser (26). Todos ellos con el objetivo de eliminar las bacterias y mantener o crear una superficie que permita la reinserción del hueso y el tejido blando.

Sin embargo, estos procedimientos demostraron ser insuficientes en la eliminación completa de la subs- 
tancia orgánica y son muchas veces responsables de contaminación adicional. Por ejemplo el láser de $\mathrm{CO}^{2}$ es potencialmente peligroso ya que la temperatura en la interfase hueso-implante alcanza los 50 grados lo que puede terminar una futura pérdida de la osteointegración según Ericsson y col 1984. Es particularmente complicado con la técnica laser hacer incidir el rayo única y exclusivamente sobre la superficie del implante, sobre todo en los casos mas comprometidos de perdida vertical de hueso periimplantario.

Sin embargo Haas y col en 1997 examinaron el efecto bactericida de irradiación con laser in contra bacterias asociadas a periimplantitis. Dicho tratamiento, permitió eliminar las bacterias presentes $(25 ; 26)$.

\section{Tratamiento de la periimplantitis mediante terapia antiinfecciosa y regeneración ósea guiada}

Una vez controlado el proceso inflamatorio en los tejidos periimplantarios, se intenta reestablecer la osteointegración utilizando procedimientos regenerativos.

Muchos estudios demuestran éxito clínico y radigráfico del tratamiento regenerativo de defectos por periimplantitis. Sin embargo no existe evidencias histológicas de una verdadera reosteointegración $(44 ; 46)$.

\section{Estudios en animales}

- Jovanovic y col 1993 (34) analizaron en perros la reparación de defectos periimplantarios inducidos por ligaduras sobre tres tipos diferentes de superficies, tales como, titanio mecanizado (implantes Branemark ), plasma spray de titanio ( IMZ) y hidroxiapatita (Integral ).Tras el tratamiento con aplicación de ácido cítrico y membrana no reasorbible de PTFE-e, la evaluación histológica permitió ver regeneración ósea en todos los implantes tratados, evidenciandose en algunas áreas un nuevo contacto entre el hueso y el implante sin interposición de tejido conectivo.

- Grunder y Col 1993 (24) no encontraron resultados positivos tras ROG de defectos en periimplantitis experimental en perros con implantes de titáneo mecanizado. En este estudio un grupo de implantes recibían membranas de PTFE-e mientras que otro grupo no recibía tratamiento regenerativo. El análisis histológico no mostró diferencias significativas, en ambos casos aparecía tejido conectivo, con poco hueso neoformado.

- Persson y Col 1996 (52) encontraron resultado negativos tras la terapia mediante ROG en defectos alrede- dor de implantes Branemark mecanizados. El estudio comparaba un tratamiento experimental mediante antibióticoterapia, desbridamiento a colgajo, destoxificación de la superfice y colocación de membranas de PTFE-e, frente al grupo control donde no se realiza ningún tratamiento. En ambos grupos se produjo cicatrización mediante tejido conectivo con ausencia de nueva osteointegración.

- Hurzeler y col 1995, 1997 (30). Compararon el tratamiento de defectos periimplantarios en perros beagle con: desbridamiento; desbridamiento y colocacion de HA reabsorbible y recubrimiento con membranas; desbridamiento, colocación de hueso liofilizado y recubrimiento con membranas. Donde se empleaba ROG aparecia mayor grado de relleno óseo, seguido de los grupos de injerto de hueso y desbridamiento a colgajo, no se encontraron diferencias entre ROG y ROG mas injerto óseo. Histológicamente los defectos tratados con HA resultaron con un mayor porcentaje de osteointegración $2,3 \mathrm{~mm}$ frente a los 2,2 del hueso liofilizado.

- Hanisch y col 1997 (28) Usando una BMP-2 (proteina osea morfogenetica) como material de relleno evaluaron el tratamiento de defectos de periimplantitis. En los defectos tratados con desbridamiento, destoxificación con ácido citrico y relleno con Rh BMP-2) el aumento del hueso fue de 2,6 mm, con regeneración de un $70 \%$ del defecto original. En el grupo control (sin BMP-2) el aumento fue de $0,8 \mathrm{~mm}$. Los autores concluyeron que la proteina morfogenetica ósea tipo 2 promueve la formación ósea y la reosteointegración en defectos experimentales de periimplantitis.

- Persson y col 2001 (51) investigaron la importancia del tipo de superficie del implante en la reosteointegracion tras periimplantitis. Examinaron el potencial de reosteointegración en tres implantes ITI con superficie SLA y tres con superficie lisa colocados en perros beagle a los que se les había inducido un proceso de periimplantitis. Tras la terapia antibiótica y desbidamiento, se sumergieron los implantes. Seis meses después se realizaron biopsias aportando la siguiente información, en los implante con superficie SLA se consiguió un relleno del $76 \%$ con un $84 \%$ de reosteointegración mientras que en los de superficie lisa se rellenaron, un $72 \%$ sus defectos con un índice del $22 \%$ de reosteointegación. Dicha información se atribuyo a la capacidad osteofílica de la superficie SLA.

-Nociti y col 2000 evaluaron la cicatrización de defectos generados por periimplantitis tras el tratamiento con ROGy/o injertos óseos. No se encontraron diferencias significativas respecto al porcentaje de contacto hueso implante entre los dos tratamientos.

-Nociti y col 2001 (48) Evaluaron clínicamente la utili- 
zación de membranas reabsorbibles y no reabsorbibles asociadas o no a injertos óseos, en el tratamiento de periimplantitis inducida por ligaduras en perros. Encontrando que no habia diferencias entre los tratamientos, con respecto al relleno óseo.

\section{Estudios en Humanos}

- Lehman y cols 1992 (38) presentaron un caso clínico de dos implantes con inflamación, supuración y profundidad de sondaje de $9 \mathrm{~mm}$ pero sin movilidad. Administraron amoxicilina $750 \mathrm{mg}$ y ornidazol 500mg durante 10 dias; instrumentaron de forma mecánica y quirúrgica y cubrieron el defecto con una membrana de PTFE-e. Cinco meses después retiraron la membrana y verificaron el relleno óseo, ganancia ósea radiográfica y mejoría en todos los parámetros clínicos.

- Hammerle y col 1995 (27) presentaron dos casos clínicos tratados con éxito, en los que se combinaba terapia antiinfecciosa y regenerativa. El tratamiento consistió en desbridamiento a colgajo, irrigación con clorhexidina y regeneración mediante membranas de PTFE-e. Al año radiográficamente se mostraba 1,5 a 3,6 $\mathrm{mm}$ de ganancia de hueso.

- Melloning y col 1995 (42) presentaron tres casos clínicos. El primero se trato mediante desbridamiento, detoxificación con tetraciclina, colocación del material de relleno y la membrana. $\mathrm{Al}$ año la profundidad de sondaje se redujo $2 \mathrm{~mm}$ y el relleno óseo radiográfico era completo. En los otros dos casos el relleno fue con hueso liofilizado y también se consiguieron mejorías favorables con respecto al relleno óseo radiográfico y a la profundidad de sondaje.

- Benheke y col 2000 (9). Utilizaron para el tratamiento de las lesiones de periimplantitis desbridamiento a

\begin{tabular}{|c|c|c|c|}
\hline \multirow{5}{*}{$\begin{array}{l}\text { CONTROL } \\
\text { (cada } \\
\text { 3-4 meses) }\end{array}$} & Negativo & \multicolumn{2}{|l|}{ Espaciar las visitas } \\
\hline & $\begin{array}{l}\text { Placa y } \\
\text { sanqrado }\end{array}$ & \multicolumn{2}{|l|}{ Pauta A } \\
\hline & \multirow{3}{*}{$\begin{array}{l}\text { Periim- } \\
\text { plantitis }\end{array}$} & $\begin{array}{l}\text { Bolsa }<5 \mathrm{~mm} \text { o pérdida } \\
\text { ósea ligera en la } \\
\text { radiografía. }\end{array}$ & Pauta A+B \\
\hline & & $\begin{array}{l}\text { Bolsa }>5 \mathrm{~mm} \text { o pérdida } \\
\text { ósea evidente en la } \\
\text { radiografía }\end{array}$ & Pauta C \\
\hline & & Corrección tisular & Pauta C+D \\
\hline
\end{tabular}

Fig. 5 colgajo y injertos oseos obtenidos de la zona retomolar o del menton,fijados con tornillos.Solo en dos de los casos de los 25 obtuvieron resultados negativos en los demás la reentrada divulgó un relleno del $90 \%$.

- Haas y col 2000 (25). Utilizaron desbridamiento a colgajo, decontaminación de la superficie con azul de toluidina, seguido de lavado con solución salina e irradiación con láser, para el tratamiento de los defectos por periimplantitis. Los defectos fueron rellenados con hueso autólogo y cubiertos con una membrana de PTFE-e.En 21 de 24 implantes se observo relleno limitado del defecto óseo ( media 21,8\%). (Fig. 5)

\section{MANTENIMIENTOY TRATAMIENTO DE PERIIMPLANTITIS CIST (fig 5)}

El CIST es un método de mantenimiento de implantes y tratamiento de periimplantitis utilizado en la universidad de Berna. El principio de este método es detectar las infecciones periimplantarias de forma temprana y utilizar el tratamiento adecuado para cada caso.La base del éxito es un sistema de visitas frecuentes para el control de:

- Presencia de placa, sangrado, supuración, bolsas periimplantarias.

- Presencia de evidencia radiográfica de pérdida ósea.

- A) Desbridamiento mecánico y refuerzo de higiene oral.

- B) Irrigación con CHX $0.2 \%$ en enjuagues $2 \mathrm{X} /$ día.

- C) Tratamiento antibiótico (Ornidazol 250 mg cada $8 \mathrm{hrs}$ ) o según análisis microbiológico y desbridamiento.

- D) Tratamiento quirúrgico (resectivo o regenerativo).

- E) Remoción del implante.

\section{CONCLUSIÓN}

Los dientes naturales pueden ser afectados por patología periodontal y los implantes dentales al mismo tiempo por periimplantitis. A pesar de que el fallo de la mayoría de los implantes dentales esta causado y originado por bacterias, Tal vez haya sutiles diferencias en la microflora y los mecanismos biológicos, y estos deben de ser mayormente estudiados para valorar su significancia clínica. 
Cabe apuntar que no existen protocolos de mantenimiento ni de tratamiento definitivos, sino que se encuentran en un proceso evolutivo y caben más estudios de investigación para su desarrollo.

\section{SUMMARY}

In this review article of periimplantitis an overview of the anatomical basic concepts are present followed by the osseointegration criteria, a study of the microbiological evidence, pathogenia, and risk factors, wich affect to the inflamatory and destructive periimplantar process. This paper continues by explaining the clinical parameters of the diseases, how to develop a diagnosis and the therapeutic and maintinance possibilities based on the scientific protocols.

\section{KEY WORDS}

Peri-implantitis. Peri-implant infection. Risk factors. Treatment of peri-implantitis

\section{CORRESPONDENCIA}

Fernando Franch Chillida

Tel: 669758251

$\mathrm{C} /$ Mayor $\mathrm{N}^{\circ} 135^{\circ} \mathrm{D}$

Madrid 28013

e-mail fernandofranch@hotmail.com

\section{BIBLIOGRAFÍA}

1. Adell R, Lekholm U, Rockler B, Branemark PI. A 15-year study of osseointegrated implants in the treatment of the edentulous jaw. Int J Oral Surg 1981; 10 (6): 387-416.

2. Alandez FJ, Lazaro PJ, Carasol M, Herrera JI, Bascones A. Características clínico histológicas de los tejidos blandos periimplantarios. Avances en Periodoncia. 1991; 3, 113-21.

3. Albrektsson T, Dahl E, Enbom L, Engevall S, Engquist B, Eriksson $A R$ et al. Osseointegrated oral implants. A Swedish multicenter study of 8139 consecutively inserted Nobelpharma implants. J Periodontol 1988; 59 (5): 287-96.

4. Albrektsson T, Isidor F. Proceedings of the first european workshop on periodontology. Consensus report session IV,. 1994. London, Quintessence. 365-9.
5. Albrektsson T, Zarb G, Worthington P, Eriksson AR. The long-term efficacy of currently used dental implants: a review and proposed criteria of success. Int J Oral Maxillofac Implants 1986; 1 (1): 11-25.

6. Apse P, Zarb GA, Schmitt A, Lewis DW. The longitudinal effectiveness of osseointegrated dental implants. The Toronto Study: peri-implant mucosal response. Int J Periodontics Restorative Dent 1991; 11 (2): 94-111.

7. Augthun M, Conrads G. Microbial findings of deep periimplant bone defects. Int J Oral Maxillofac Implants 1997; 12 (1): 106-12.

8. Bain CA, Moy PK. The association between the failure of dental implants and cigarette smoking. Int J Oral Maxillofac Implants 1993; 8 (6): 609-15.

9. Behneke A, Behneke N, d'Hoedt B. Treatment of periimplantitis defects with autogenous bone grafts: sixmonth to 3-year results of a prospective study in 17 patients. Int J Oral Maxillofac Implants 2000; 15 (1): 12538.

10. Berglundh T, Lindhe J, Ericsson I, Marinello CP, Liljenberg B, Thomsen P. The soft tissue barrier at implants and teeth. Clin Oral Implants Res 1991;2 (2): 8190.

11. Berglundh T, Lindhe J, Marinello C, Ericsson I, Liljenberg B. Soft tissue reaction to de novo plaque formation on implants and teeth. An experimental study in the dog. Clin Oral Implants Res 1992; 3 (1): 1-8.

12. Bower RC, Radny NR, Wall CD, Henry PJ. Clinical and microscopic findings in edentulous patients 3 years after incorporation of osseointegrated implant-supported bridgework. J Clin Periodontol 1989; 16 (9): 580-7.

13. Buser D, Mericske-Stern R, Bernard JP, Behneke A, Behneke N, Hirt HP et al. Long-term evaluation of nonsubmerged ITI implants. Part 1: 8-year life table analysis of a prospective multi-center study with 2359 implants. Clin Oral Implants Res 1997; 8 (3): 161-72.

14. Buser D, Weber HP, Bragger U, Balsiger C. Tissue integration of one-stage ITI implants: 3-year results of a longitudinal study with Hollow-Cylinder and Hollow-Screw implants. Int J Oral Maxillofac Implants 1991; 6 (4): 40512.

15. Buser D, Weber HP, Lang NP. Tissue integration of nonsubmerged implants. 1-year results of a prospective study with 100 ITI hollow-cylinder and hollow-screw implants. Clin Oral Implants Res 1990; 1 (1): 33-40.

16. Carmichael RP, Apse P, Zarb GA, Mc-Cullogh AG. Biological,Microbiological and clinical aspcts of the 
periimplant mucosa. The Branemark Osseointegrated implant. Chicago: Quintaessence, 1989.

17. Ciancio SG, Lauciello F, Shibly O,Vitello M, Mather M. The effect of an antiseptic mouthrinse on implant maintenance: plaque and peri-implant gingival tissues. J Periodontol 1995; 66 (11): 962-5.

18. Delgado E, Sanchez MA, Romeu J, Berini L, Gay C. Enfermedad periimplantaria. Etiologia Fisiopatologia y diagnóstico. Revisión de la literatura. Archivos de odontoestomatologia 1999; 15 (2): 53-67.

19. Ellegaard B, Baelum V, Karring T. Implant therapy in periodontally compromised patients. Clin Oral Implants Res 1997; 8 (3): 180-8.

20. Ericsson I, Lindhe J. Probing depth at implants and teeth. An experimental study in the dog. J Clin Periodontol $1993 ; 20$ (9): 623-7.

21. Ericsson I, Persson LG, Berglundh T, Edlund T, Lindhe J. The effect of antimicrobial therapy on periimplantitis lesions. An experimental study in the dog. Clin Oral Implants Res 1996; 7 (4): 320-8.

22. Esposito M, Hirsch JM, Lekholm U, Thomsen P. Biological factors contributing to failures of osseointegrated oral implants. (II). Etiopathogenesis. Eur J Oral Sci 1998; 106 (3): 721-64.

23. Fardal O, Johannessen AC, Olsen I. Severe, rapidly progressing peri-implantitis. J Clin Periodontol 1999; 26 (5): 313-7.

24. Grunder U, Hurzeler MB, Schupbach P, Strub JR. Treatment of ligature-induced peri-implantitis using guided tissue regeneration: a clinical and histologic study in the beagle dog. Int J Oral Maxillofac Implants 1993; 8 (3): 282-93.

25. Haas R, Baron M, Dortbudak O, Watzek G. Lethal photosensitization, autogenous bone, and e-PTFE membrane for the treatment of peri-implantitis: preliminary results. Int J Oral Maxillofac Implants 2000; 15 (3): 374-82.

26. Haas R, Dortbudak O, Mensdorff-Pouilly N, Mailath G. Elimination of bacteria on different implant surfaces through photosensitization and soft laser. An in vitro study. Clin Oral Implants Res 1997; 8 (4): 249-54.

27. Hammerele C.H., Fourmousis I, Winkler J.R., Weigel C, Bragger U, Lang N.P. Succesful bone fill in late preiimplant defects using guide tissue regeneration. a short comunication. J periodontology 1995; 66: 303-8.

28. Hanisch O, Tatakis DN, Boskovic MM, Rohrer MD, Wikesjo UM. Bone formation and reosseointegration in peri- implantitis defects following surgical implantation of rhBMP-2. Int J Oral Maxillofac Implants 1997; 12 (5): $604-$ 10 .

29. Hultin M, Gustafsson A, Klinge B. Long-term evaluation of osseointegrated dental implants in the treatment of partly edentulous patients. J Clin Periodontol 2000; 27 (2): 128-33.

30. Hurzeler MB, Quinones CR, Morrison EC, Caffesse RG. Treatment of peri-implantitis using guided bone regeneration and bone grafts, alone or in combination, in beagle dogs. Part 1: Clinical findings and histologic observations. Int J Oral Maxillofac Implants 1995; 10 (4): 474-84.

31. Isidor F. Loss of osseointegration caused by occlusal load of oral implants. A clinical and radiographic study in monkeys. Clin Oral Implants Res 1996; 7 (2): 143-52.

32. Jaffin RA, Berman CL. The excessive loss of Branemark fixtures in type IV bone: a 5-year analysis. J Periodontol $1991 ; 62(1): 2-4$

33. Jemt T, Lekholm U. Oral implant treatment in posterior partially edentulous jaws: a 5-year follow-up report. Int J Oral Maxillofac Implants 1993; 8 (6): 635-40.

34. Jovanovic SA, Kenney EB, Carranza FA, Donath $\mathrm{K}$. The regenerative potential of plaque induced prei-implant bone defects treated by submerged membrane technique: an experimental study. Int J Oral Maxillofac Implants 1993; 8:13-8.

35. Lang NP, Bragger U, Walther D, Beamer B, Kornman KS. Ligature-induced peri-implant infection in cynomolgus monkeys. I. Clinical and radiographic findings. Clin Oral Implants Res 1993; 4 (1): 2-11.

36. Lang NP, Wilson TG, Corbet EF. Biological complications with dental implants: their prevention, diagnosis and treatment. Clin Oral Implants Res 2000; 11 Suppl 1: 146-55: 146-55.

37. Lavigne SE, Krust-Bray KS, Williams KB, Killoy WJ, Theisen F. Effects of subgingival irrigation with chlorhexidine on the periodontal status of patients with HA-coated integral dental implants. Int J Oral Maxillofac Implants 1994; 9 (2): 156-62.

38. Lehmann B, Bragger U, Hammerle CH, Fourmousis I, Lang NP. Treatment of an early implant failure according to the principles of guided tissue regeneration (GTR). Clin Oral Implants Res 1992; 3 (1): 42-8.

39. Lindquist LW, Rockler B, Carlsson GE. Bone resorption around fixtures in edentulous patients treated with mandibular fixed tissue-integrated prostheses. J Prosthet Dent 1988; 59(1):59-63. 
40. Malmstrom HS, Fritz ME, Timmis DP, Van Dyke TE. Osseointegrated implant treatment of a patient with rapidly progressive periodontitis. A case report. J Periodontol 1990; 61 (5): 300-4.

41. Martin-Granizo R, De Pedro M, Fracasos y complicaciones en la implantología dental:¿como evitarlos? Rev Esp de cirugía Oral y Maxilofacial 2001; 23: 182-92.

42. Mellonig JT, Griffiths G, Mathys E, Spitznagel J, Jr. Treatment of the failing implant: case reports. Int J Periodontics Restorative Dent 1995; 15 (4): 384-95.

43. Mombelli A. Etiology, diagnosis, and treatment considerations in peri-implantitis. Curr Opin Periodontol 1997; 4: 127-36:127-36.

44. Mombelli A. Microbiology and antimicrobial therapy of peri-implantitis. Periodontol 2000; \%2002; 28: 177-89.: 177-89.

45. Mombelli A, Lang NP. Antimicrobial treatment of periimplant infections. Clin Oral Implants Res 1992; 3 (4): 162-8.

46. Mombelli A, Lang NP. The diagnosis and treatment of peri-implantitis. Periodontol 2000; 1998 Jun; 17: 63-76.

47. Nevins M, Langer B. The successful application of osseointegrated implants to the posterior jaw: a long-term retrospective study. Int J Oral Maxillofac Implants 1993; 8 (4): 428-32.

48. Nociti FH, Jr., Machado MA, Stefani CM, Sallum EA. Absorbable versus nonabsorbable membranes and bone grafts in the treatment of ligature-induced periimplantitis defects in dogs: a histometric investigation. Int J Oral Maxillofac Implants 2001; 16 (5): 646-52.

49. Ortega J, Bowen A, Carmona J, Benet F, Gonzalez de la Vega A. Patología periimplantaria. Gaceta Dental 2002; 125: 88-132.

50. Persson LG, Araujo MG, Berglundh T, Grondahl K, Lindhe J. Resolution of peri-implantitis following treatment. An experimental study in the dog. Clin Oral Implants Res 1999; 10 (3): 195-203.

51. Persson LG, Berglundh T, Lindhe J, Sennerby L. Re-osseointegration after treatment of peri-implantitis at different implant surfaces. An experimental study in the dog. Clin Oral Implants Res 2001; 12 (6): 595-603.

52. Persson LG, Ericsson I, Berglundh T, Lindhe J. Guided bone regeneration in the treatment of periimplantitis. Clin Oral Implants Res 1996; 7 (4): 366-72.

53. Piattelli A, Scarano A, Balleri P, Favero GA. Clinical and histologic evaluation of an active \&quot;implant periapical lesion\&quot; a case report. Int J Oral Maxillofac Implants 1998; 13 (5): 713-6.

54. Piattelli A, Scarano A, Piattelli M. Abscess formation around the apex of a maxillary root form implant: clinical and microscopical aspects. A case report. J Periodontol 1995; 66 (10): 899-903.

55. Piattelli A, Scarano A, Piattelli M, Podda G. Implant periapical lesions: clinical, histologic, and histochemical aspects. A case report. Int J Periodontics Restorative Dent 1998; 18 (2): 181-7.

56. Pontoriero R, Tonelli MP, Carnevale G, Mombelli A, Nyman SR, Lang NP. Experimentally induced periimplant mucositis. A clinical study in humans. Clin Oral Implants Res 1994; 5 (4): 254-9.

57. Quinteros M, Delgado E, Sanchez MA, Berini L, Gay C. Estudio microbiológico de la periimplantitis: Presentación de 9 casos clínicos. Avances en Periodoncia 2000; 13 (3): 137-50.

58. Quirynen M, De Soete M, van Steenberghe D. Infectious risks for oral implants: a review of the literature. Clin Oral Implants Res 2002; 13 (1): 1-19.

59. Quirynen M, van der Mei HC, Bollen CM, Schotte A, Marechal M, Doornbusch GI et al. An in vivo study of the influence of the surface roughness of implants on the microbiology of supra- and subgingival plaque. J Dent Res 1993; 72 (9): 1304-9.

60. Sanz M, Newman MG, Nachnani S, Holt R, Stewart R, Flemmig T. Characterization of the subgingival microbial flora around endosteal sapphire dental implants in partially edentulous patients. Int J Oral Maxillofac Implants 1990; 5 (3): 247-53.

61. Schenk G, Flemmig TF, Betz T, Reuther J, Klaiber B. Controlled local delivery of tetracycline $\mathrm{HCl}$ in the treatment of periimplant mucosal hyperplasia and mucositis. A controlled case series. Clin Oral Implants Res 1997; 8 (5): 427-33.

62. Shibli JA, Andrade RA, Marcantonio E. Aspectos microbiológicos de la periimplantitis. Periodoncia 2002; 12: 29-38.

63. Sussman HI, Moss SS. Localized osteomyelitis secondary to endodontic-implant pathosis. A case report. J Periodontol 1993; 64 (4): 306-10.

64. Tillmanns HW, Hermann JS, Tiffee JC, Burgess AV, Meffert RM. Evaluation of three different dental implants in ligature-induced peri-implantitis in the beagle dog. Part II. Histology and microbiology. Int J Oral Maxillofac Implants 1998; 13 (1): 59-68. 